\title{
MODELAGEM UNIDIMENSIONAL DA CAMADA LIMITE OCEÂNICA DURANTE A PASSAGEM DE UMA FRENTE FRIA NA REGIÃO DA CONFLUÊNCIA BRASIL-MALVINAS
}

\author{
DENIS HARLEY CARDOSO, MARCELO DOURADO
}

\author{
Universidade Federal do Paraná (UFPR), Curitiba, PR, Brasil \\ deniscardoso@ufpr.br,dourado@ufpr.br
}

Recebido Outubro de 2012 - Aceito Novembro de 2013

\begin{abstract}
RESUMO
Um modelo unidimensional com fechamento de segunda ordem é usado para investigar a resposta da superfície do oceano à passagem de uma frente fria na região da Confluência Brasil-Malvinas. Dados da boia ARGOS-32056 foram utilizados para forçar e validar o modelo para o período entre $12 \mathrm{e}$ 20 de Janeiro de 2002, correspondente à ação de uma frente fria. O modelo foi capaz de reproduzir tanto o ciclo diurno, quanto o resfriamento da temperatura da superfície do mar observado com a passagem da frente fria. Os resultados da simulação numérica mostraram um aumento de $0,2{ }^{\circ} \mathrm{C}$ no efeito do resfriamento de pele e um aprofundamento de aproximadamente 16 metros na camada de mistura oceânica com a passagem da frente fria. Essas variações foram consistentes com uma mudança no balanço de energia em superfície, devido ao aumento abrupto dos fluxos turbulentos em resposta ao aumento da velocidade do vento e da diferença de temperatura entre o ar e o mar. Após a passagem da frente fria, os fluxos de calor diminuem lentamente mostrando que, se não houver a entrada de uma nova frente fria ou advecção de massa de água, a superfície do mar e o ar adjacente tendem ao equilíbrio.
\end{abstract}

Palavras-chave: Modelo unidimensional, camada de mistura oceânica, sistema frontal.

ABSTRACT: ONE-DIMENSIONAL MODELING OF THE OCEANIC BOUNDARY LAYER DURING PASSAGE OF A COLD FRONT IN THE BRAZIL-MALVINAS CONFLUENCE REGION A one-dimensional second order closure model is used to investigate the response of the ocean surface to the passage of a cold front in the region of the Brazil-Malvinas Confluence. In order to initialize, force and validate the model, data from a moored buoy ARGOS-23056, for the period between 12 and 20 January 2002 were used, corresponding to the passage of a cold front. The model was able to reproduce both the diurnal cycle and the cooling surface temperature observed with the passage of the cold front. Numerical simulation results showed an increased of $0.2^{\circ} \mathrm{C}$ in the cool skin effect and a deepening of approximately 16 meters in the oceanic mixed layer with the passage of the cold front. These variations were consistent with a change in surface energy balance, due to an abrupt increase in turbulent fluxes in response to the increased wind speed and the temperature difference between the air and sea. After the cold front passage, the heat fluxes decreased slowly, showing that if, there is no passage of another cold front neither water advection, the ocean surface and the air adjacent tend towards equilibrium.

Keywords: One-dimensional model, oceanic mixed layer, frontal system. 


\section{INTRODUÇÃO}

A investigação dos processos de interação do oceano com a atmosfera é importante para o entendimento dos sistemas de tempo e clima. Isto porque os processos turbulentos que ocorrem na interface ar-mar, em pequenas escalas espaciais e temporais, influenciam nos processos meteorológicos e oceânicos de grande escala (Dourado e Caniaux, 2003). Além disto, a compreensão de como as Camadas Limite Oceânica (CLO) e Atmosférica (CLA) interagem é fundamental para melhorar a previsão numérica do tempo e do clima (Weng e Taylor, 2003). A turbulência nessa interface pode ser intensificada em eventos de frente fria, desempenhando um papel importante nas trocas de calor, momentum e massa entre a superfície do oceano e a camada atmosférica adjacente (Dourado e Oliveira, 2008).

A ocorrência de frentes frias é comum durante todo o ano no Brasil, principalmente nas regiões Sul e Sudeste (Rodrigues et al., 2004). Estes sistemas além de alterarem os campos de vento, pressão, temperatura e outras variáveis atmosféricas durante a sua trajetória, interagem também com a superfície do mar (Wallace e Hobbs, 2006). Alguns estudos investigaram a influência da passagem de uma frente fria sobre a superfície do mar.

Xue et al. (2000) utilizaram modelagem acoplada oceano-atmosfera para estudar as interações ar-mar durante a advecção de uma massa de ar frio sobre a Corrente do Golfo. $\mathrm{O}$ estudo mostrou que o aumento da velocidade do vento com a passagem do sistema tem um impacto considerável sobre o oceano, e em sua resposta para a atmosfera via fluxos de superfície. Dourado e Oliveira (2001) investigaram as CLO e CLA sobre a região de Cabo Frio durante a passagem de um sistema frontal. Um aumento na extensão vertical de ambas as camadas foi observado com uma associada intensificação da mistura mecânica, devido principalmente, ao aumento da velocidade do vento em superfície.

Persson et al. (2005) analisaram dados da camada atmosférica superficial, obtidos durante o Fronts and Atlantic Storm Tracks Experiment (FASTEX), para avaliar processos de interação ar-mar durante a passagem de 10 sistemas frontais. Os autores mostraram que o fluxo turbulento de momentum é máximo exatamente após a passagem do sistema frontal, durante o pico de velocidade do vento em baixos níveis. Os fluxos de calor latente e sensível são mínimos exatamente após a passagem do sistema frontal, a despeito dos fortes ventos, devido à umidificação e aquecimento associado aos padrões de escala sinótica.

Mais recentemente, Dourado e Oliveira (2008) utilizaram um modelo atmosférico unidimensional acoplado a um modelo de camada de mistura oceânica, para investigar o impacto dos contrastes térmicos entre o oceano e a atmosfera na estrutura vertical da CLA e CLO na região de Cabo Frio. Para isto, foram realizadas simulações a partir de dados observacionais obtidos durante a passagem de uma frente fria sobre a região. Os resultados indicaram que, para a atmosfera, o termo de produção mecânica é predominante e que o contraste térmico tem um grande impacto na evolução temporal da profundidade da camada de mistura. Esta apresentou um aprofundamento controlado pela convecção térmica na CLA associada à interrupção do regime de ressurgência e à passagem da frente fria.

No Oceano Atlântico Sudoeste, uma área importante no que diz respeito às interações ar-mar é a região da convergência Brasil-Malvinas. Esta área é caracterizada pelo intenso gradiente horizontal de temperatura, devido aos meandros e vórtices oceânicos gerados pelo encontro das águas quentes e frias das correntes do Brasil e das Malvinas, respectivamente. O contraste térmico entre estas correntes provoca a intensificação dos fluxos de calor nas massas de ar frio que se deslocam do continente para o mar. Além disso, a liberação de calor latente tem um papel importante no balanço energético da ciclogênese nessa região (Pezzi et al., 2005).

Em estudos de modelagem numérica, dois tipos de modelos fundamentalmente distintos são utilizados para simular o comportamento da CLO sob condições forçadas: modelos de camada de mistura (bulk, slab models) e modelos de difusão. Os modelos de camada de mistura tentam reproduzir o comportamento da camada limite planetária (CLP) num sentido integral. Eles assumem, a priori, a existência de uma camada bem misturada. Já nos modelos de difusão, as propriedades da CLP são diretamente parametrizadas como uma função da mistura turbulenta. Eles se baseiam na modelagem das quantidades turbulentas, tais como, os momentos de segunda (Mellor e Yamada, 1974,1982; Gaspar et al., 1990, D’Alessio et al., 1998) ou terceira ordem (André e Lacarrère, 1985). A maior vantagem desta abordagem mais completa é que eles incorporam mais informação física em comparação aos fechamentos de ordem inferiores (Khanta e Clayson, 1994). Além disto, o modelo de fechamento unidimensional de segunda ordem fornece mais informações a um custo computacional baixo.

Neste artigo foi investigada a influência da passagem de uma frente fria sobre a superfície oceânica na região da confluência Brasil-Malvinas. Para tal, um modelo unidimensional de fechamento de segunda ordem baseado em Mellor e Yamada (1982) foi utilizado para simular numericamente a camada limite oceânica forçada sob condições de frente fria. Os modelos de fechamento de segunda ordem do tipo Mellor e Yamada (M-Y) são extensivamente usados para simular a camada limite oceânica e atmosférica.

Este artigo está organizado da seguinte maneira. $\mathrm{Na}$ seção 2 , as principais características do modelo bem como a implementação numérica do experimento são apresentadas. Os 
resultados são apresentados e discutidos na seção 3 e, na seção 4 são apresentadas as conclusões.

\section{MATERIAL E MÉTODOS}

\subsection{Descrição do modelo}

O modelo de fechamento de segunda ordem usado aqui foi baseado na versão 2.5 de Mellor e Yamada (1982), desenvolvido por Dourado e Oliveira (2001). Este consiste em resolver as equações para os momentos de segunda ordem obtidas das equações de Reynolds do movimento e da termodinâmica, fechando-as através de parametrizações dos momentos de terceira ordem. A homogeneidade horizontal é assumida, de modo que todas as propriedades da CLO são apenas função da coordenada vertical z e do tempo t. Assim, considerando que o oceano na camada limite oceânica satisfaz as condições de Boussinesq, as equações para as quantidades médias resultantes assumem a seguinte forma:

$$
\begin{aligned}
& \frac{\partial \bar{u}}{\partial t}=-f \bar{v}-\frac{\partial \overline{u^{\prime} w^{\prime}}}{\partial z} \\
& \frac{\partial \bar{v}}{\partial t}=f \bar{u}-\frac{\partial \overline{v^{\prime} w^{\prime}}}{\partial z} \\
& \frac{\partial \bar{T}}{\partial t}=-\frac{\partial \overline{T^{\prime} w^{\prime}}}{\partial z}-\frac{\partial R_{n}}{\partial z} \\
& \frac{\partial \bar{S}}{\partial t}=-\frac{\partial \overline{S^{\prime} w^{\prime}}}{\partial z}
\end{aligned}
$$

onde $\bar{u} \mathrm{e} \bar{v}$, são as componentes zonal e meridional da velocidade, $\mathrm{f}=2 \Omega$ sen $\theta$ é o parâmetro de Coriolis, sendo $\Omega$ a velocidade angular da terra $\left(7,292 \times 10^{-5} \mathrm{rad} \mathrm{s}^{-1}\right)$ e $\theta$ a latitude, $\bar{T}$ é a temperatura do mar, $R n$ é o fluxo vertical médio de radiação líquida, e $\bar{S}$ é a salinidade. As covariâncias entre as componentes horizontais da velocidade ( $\left.\overline{u^{\prime} w^{\prime}} \mathrm{e} \overline{v^{\prime} w^{\prime}}\right)$, temperatura da água do mar $\left(\overline{T^{\prime} w^{\prime}}\right)$, salinidade $\left(\overline{S^{\prime} w^{\prime}}\right)$ e componente vertical da velocidade, correspondem aos fluxos turbulentos verticais de momentum, temperatura e sal na camada limite oceânica. O sistema de equações acima é composto por 4 equações e 8 variáveis desconhecidas, sendo quatro momentos estatísticos de primeira ordem $(\bar{u}, \bar{v}, \bar{T}, \bar{S})$ e quatro de segunda ordem $\left(\overline{u^{\prime} w^{\prime}}, \overline{v^{\prime} w^{\prime}}, \overline{T^{\prime} w^{\prime}}, \overline{S^{\prime} w^{\prime}}\right)$. O sistema é completado pela inclusão de 10 equações para obter a variância $\overline{\left(u^{\prime} u^{\prime}\right.}, \overline{v^{\prime} v^{\prime}}, \overline{w^{\prime} w}, \overline{T^{\prime} T^{\prime}}$, $\overline{\left.S^{\prime} S^{\prime}\right)}$ e covariância $\left(\overline{T^{\prime} u^{\prime}}, \overline{T^{\prime} v^{\prime}}, \overline{S^{\prime} u^{\prime}}, \overline{S^{\prime} v^{\prime}}, \overline{T^{\prime} S^{\prime}}\right)$, em termos de quantidades médias.

Assim, o sistema resultante não é fechado, visto que é formado por 14 equações e um número maior de variáveis desconhecidas. Desse modo, o problema de fechamento é resolvido através da parametrização dos termos desconhecidos em função dos momentos estatísticos de primeira e segunda ordem (Mellor e Yamada, 1982). As escalas de comprimento usadas neste modelo de fechamento são estimadas pela versão modificada da expressão de Blackadar (Blackadar, 1962) proposto por Mellor e Yamada (1982).

As condições de contorno superior são dadas por:

$$
\begin{aligned}
& -\left.\rho_{a} c_{p} \overline{T^{\prime} w^{\prime}}\right|_{o}=F_{\text {nsol }}=H+L E+I R \\
& -\left.\rho_{a} c_{p} \overline{S^{\prime} w^{\prime}}\right|_{o}=E-P \\
& -\left.\rho_{a} \overline{u^{\prime} w^{\prime}}\right|_{o}=\tau_{x} \\
& -\left.\rho_{a} \overline{v^{\prime} w^{\prime}}\right|_{o}=\tau_{y}
\end{aligned}
$$

onde $\mathrm{F}_{\text {nsol }}$ representa o fluxo não solar, dado pela soma dos fluxos de calor sensível $(H)$, calor latente $(L E)$, e infravermelho $(I R) ; E$ e $P$ são as taxas de evaporação e precipitação, respectivamente; $\rho_{a}$ é a densidade do ar; $c_{p}$ é o calor específico à pressão constante do ar; $\tau_{x}$ e $\tau_{y}$ são as componentes zonal e meridional do stress do vento à superfície, respectivamente. Os fluxos turbulentos foram estimados utilizando o esquema bulk COARE 3.0 proposto por Fairall et al. (2003).

\subsection{Experimento numérico}

Os dados meteorológicos e oceanográficos utilizados neste trabalho são provenientes da boia de fundeio ARGOS32056, e são disponibilizados pelo Programa Nacional de Boias (PNBOIA), que faz parte da contribuição brasileira para o Global Ocean Observation System (GOOS). Para identificar o caso frontal foram utilizados os dados da boia, bem como, cartas sinóticas de superfície fornecidas pelo Departamento de Hidrologia e Navegação DHN/Marinha.

O período escolhido para este estudo é o de 12 a 20 de Janeiro de 2002, caracterizado pela entrada de uma frente fria no dia 14 (Figura 1), com a permanência do vento de quadrante sul até o dia 18. Os dias 12 e 13 de Janeiro compreendem o período pré-frontal, caracterizado por ventos de direção predominante Norte-Nordeste, variando entre $7 \mathrm{~m} / \mathrm{s}$ e $10 \mathrm{~m} / \mathrm{s}$ (Figura 2). A temperatura do ar neste período oscilou entre $24,5^{\circ} \mathrm{C}$ e $25^{\circ} \mathrm{C}$, e não apresentou variações significativas. $\mathrm{O}$ mesmo foi observado para a umidade relativa no período pré-frontal, que se manteve acima de $80 \%$ e atingiu valores de $90 \%$.

No dia da passagem da frente fria (dia 14 de Janeiro) houve um aumento da ordem de $11 \mathrm{~m} / \mathrm{s}$ na velocidade do vento, que atingiu um pico de $15 \mathrm{~m} / \mathrm{s}$, bem como a virada de sua direção para o quadrante Sul. Além disso, com a passagem do sistema frontal foi observada também uma redução da temperatura e umidade do ar da ordem de $2^{\circ} \mathrm{Ce} 20 \%$, respectivamente, (Figura 

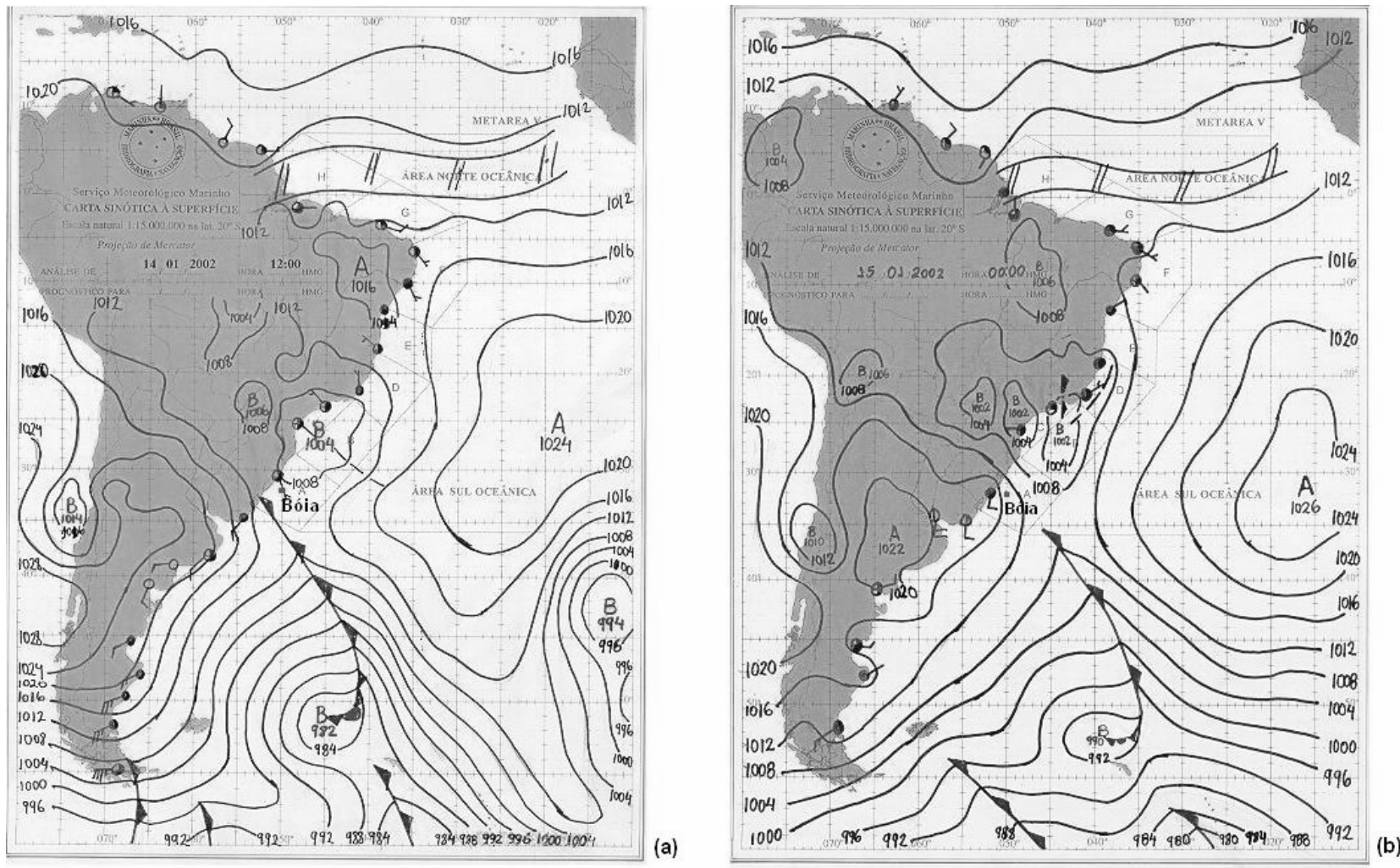

Figura 1 - Cartas sinóticas de pressão ao nível do mar (hPa) para (a) 14/01/02 às 12 UTC; (b) 15/01/02 às 00 UTC. FONTE: DHN - MARINHA DO BRASIL

(a) Temperatura do ar

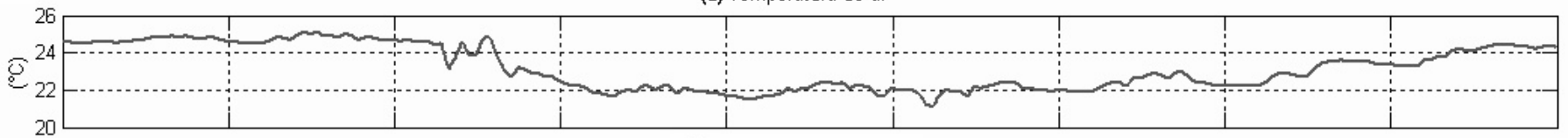

(b) Umidade relativa do ar
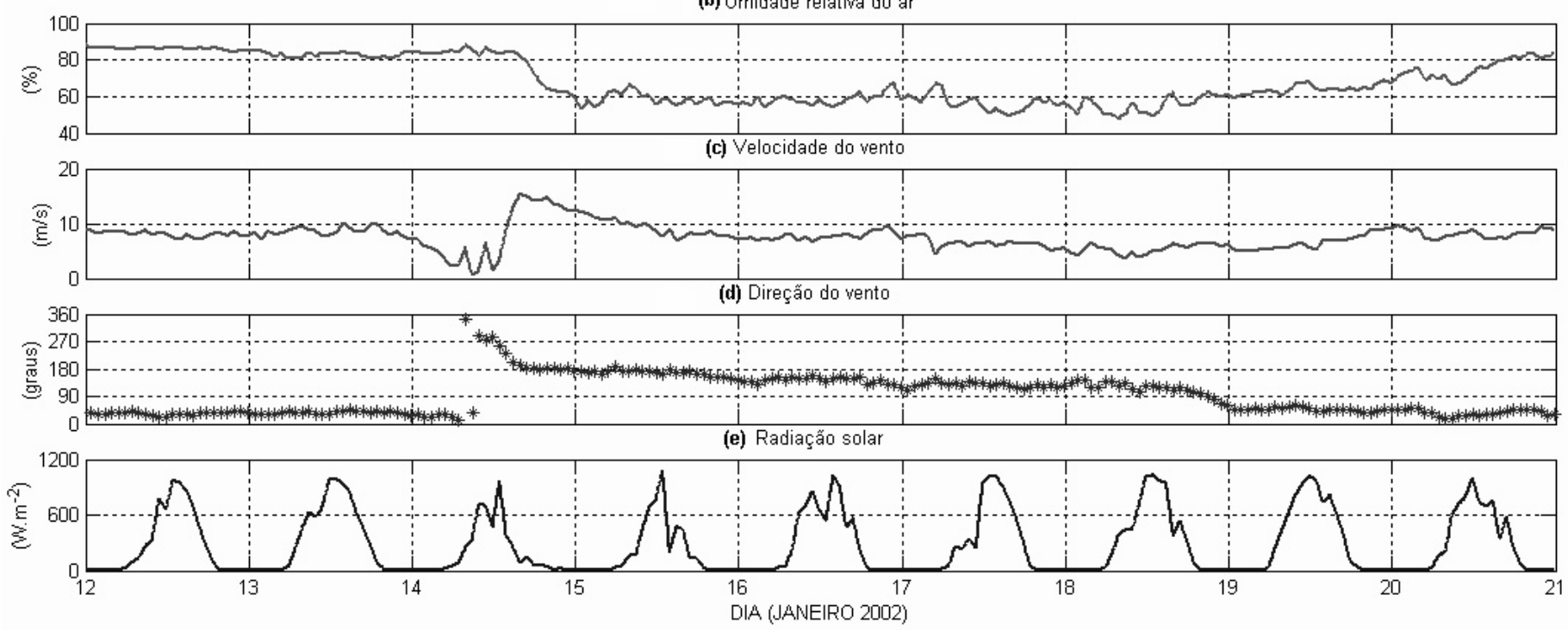

Figura 2 - Evolução temporal da (a) temperatura do ar, (b) umidade relativa (c) velocidade do vento (d) direção do vento e (e) radiação solar, observados para o período de estudo. 
2) associada à entrada da massa de ar frio e seco localizado na retaguarda do sistema frontal.

Após a passagem da frente fria no período pós-frontal (15 a 20 de Janeiro), os valores da temperatura e umidade relativa foram menores em relação ao pré-frontal, com a temperatura variando entre $21^{\circ} \mathrm{C}$ e $24,5^{\circ} \mathrm{C}$, e a umidade entre $50 \%$ e $80 \%$ até o dia 20 de Janeiro. A influência do sistema se deu até o dia 18, caracterizado pela virada do fluxo do vento para o quadrante Norte, com direções predominantes de Nordeste que se mantiveram até o final do período. Antes desse dia a velocidade do vento oscilou entre 5 e $10 \mathrm{~m} / \mathrm{s}$ aproximadamente, e houve o predomínio de ventos de Sul-Sudeste.

A simulação numérica para o período de estudo foi realizada em um tempo de integração de 9 dias (dia 12 a 20 de Janeiro de 2002), com um passo de tempo de 5 minutos. A resolução vertical utilizada foi de 1 metro, com 101 níveis de profundidade desde a superfície $(0,5 \mathrm{~m})$ até o nível máximo próximo ao fundo $(100,5 \mathrm{~m})$.

\subsection{Condições iniciais e forçantes de superfície}

Como condições iniciais para a integração do modelo foram utilizados perfis verticais da temperatura do mar e salinidade. Os perfis foram construídos a partir dos valores de superfície observados para o primeiro dia e hora da simulação, relacionando-os com dados climatológicos do World Ocean Atlas e National Oceanographic Data Center (NOAA), bem como dados da literatura que tratam da temperatura do mar e salinidade para a região (Piola et al., 2000; Piola e Matano, 2001; Soares e Möller, 2001, Silveira et al., 2000).

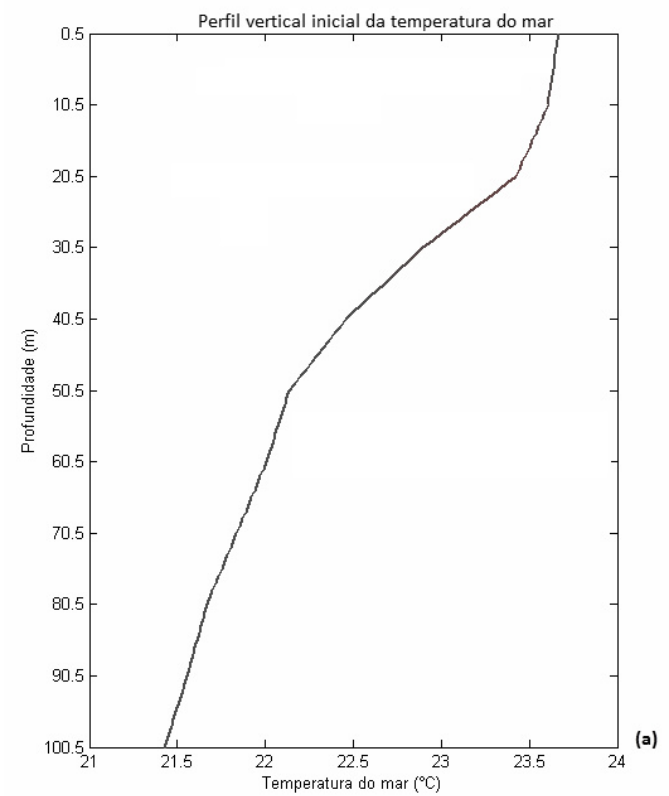

No caso do perfil inicial da temperatura do mar (Figura $3 a)$, assumiu-se a presença de uma primeira camada superficial bem misturada até os $20 \mathrm{~m}$ de profundidade, com valores próximos ao valor inicial da TSM de $23,6{ }^{\circ} \mathrm{C}$. Este valor está em concordância com as temperaturas encontradas em águas tropicais carreadas pela Corrente do Brasil, que de acordo com Silveira et al. (2000), apresentam temperaturas superiores a $20^{\circ} \mathrm{C}$ e salinidade maior que 36 . Assim, as principais variações assumidas aqui para o perfil da temperatura seguiram as características típicas de águas tropicais (Silveira et al., 2000; Piola e Matano, 2001; Soares e Möller, 2001). Abaixo da camada superficial bem misturada foi definida uma termoclina sazonal, com valores diminuindo até $22^{\circ} \mathrm{C}$ e limitados abaixo por uma camada relativamente homogênea até o nível máximo de 100,5 $\mathrm{m}$ de profundidade.

Para o perfil inicial da salinidade (Figura 3b), também foram consideradas as características típicas das águas tropicais carreadas pela Corrente do Brasil, somado ao efeito da presença de águas superficiais menos salinas provenientes da Lagoa dos Patos e do próprio estuário do Rio da Prata (Piola et al., 2000; Soares e Möller, 2001). Assim, devido a essa influência do aporte continental, os valores da salinidade no perfil aumentam com a profundidade. Na superfície assumiu-se uma primeira camada superficial bem misturada, com valores da ordem de 34 , limitada abaixo por uma relativa queda nos valores e pela presença de uma camada homogênea que se estende desde os 30,5 m até o nível máximo de profundidade, com valores chegando a 36,8 .

Além dos perfis iniciais de temperatura e salinidade, as componentes zonal e meridional da corrente horizontal

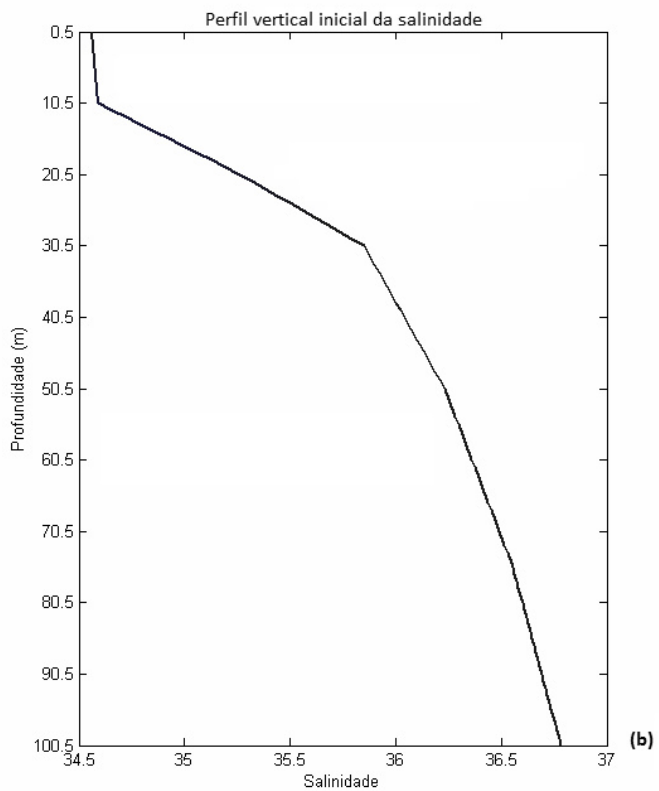

Figura 3 - Perfis iniciais da (a) temperatura do mar e (b) salinidade para o primeiro dia e hora da simulação (dia 12 de junho de 2002, às 0:00h) 
foram definidas iguais a zero no início da simulação, e foram ajustadas rapidamente em resposta ao stress do vento, conforme o avanço do experimento numérico. Os dados meteorológicos, medidos pela boia, foram utilizados como forçantes externas de superfície a cada passo de integração do modelo e, para isto, eles foram linearmente interpolados em intervalos de 5 minutos.

\section{RESULTADOS E DISCUSSÃO}

\subsection{Temperatura da superfície do mar}

A Figura 4 apresenta a evolução temporal da TSM modelada e a observada para o período de estudo. O modelo reproduziu de maneira satisfatória o ciclo diurno, com variações de até $1,3^{\circ} \mathrm{C}$ e a queda de aproximadamente $1^{\circ} \mathrm{C}$ devido à passagem da frente fria. O modelo também reproduziu, embora de maneira mais significativa, a recuperação da TSM observada após a entrada da frente. As diferenças observadas no início e fim da simulação, podem estar associados à advecção e/ou à erros no modelo.

O ciclo diário da TSM é dado pela alternância entre o aquecimento diurno devido à radiação solar incidente $\mathrm{e}$ o resfriamento noturno induzido pelos fluxos de energia direcionados do mar para o ar, superando os fluxos de entrada. Já o resfriamento associado à passagem frontal se deu pela intensificação dos fluxos turbulentos, em virtude do aumento da velocidade do vento em superfície e da diferença de temperatura entre o ar e o mar (Webster e Lukas, 1992).

Esta diminuição da TSM com a passagem da frente fria também foi observada por Xue et al. (2000), que investigaram numericamente as interações ar-mar durante a advecção de uma massa de ar frio sobre a Corrente do Golfo. Eles observaram uma redução de $1^{\circ} \mathrm{C}$ na TSM e um aprofundamento da ordem de $40 \mathrm{~m}$ na camada de mistura com a entrada do sistema frontal. De acordo com os autores, estas modificações estiveram associadas às perdas de calor do oceano superficial para atmosfera, que foram intensificadas com a passagem da massa de ar frio e seco.

\subsection{Profundidade da camada de mistura oceânica}

Neste estudo a profundidade da camada de mistura foi calculada por um critério baseado na diferença de densidade, de acordo com Sprintall e Tomczak (1992). Sua espessura foi estimada como sendo a profundidade onde a densidade é igual à da água de superfície, adicionada de um incremento $0,5^{\circ} \mathrm{C}$ na temperatura. Este mesmo intervalo foi definido para estimar a camada isotérmica, considerando a profundidade que a temperatura difere em $0,5^{\circ} \mathrm{C}$ do seu valor de superfície. Por sua vez, a camada isohalina foi calculada por um método baseado em um gradiente máximo de 0,013 psu. ${ }^{-1}$ na salinidade, que determinou a base desta camada (Anderson et al., 1996).

A profundidade da camada de mistura oceânica (CMO) estimada apresentou, para todo o período, ciclos diurnos bem marcados de até $10 \mathrm{~m}$, seguindo a oscilação diária da TSM (Figura 5). Em resposta ao aquecimento diurno, para todos os dias houve uma retração da CMO devido à diminuição da densidade na camada superior do oceano e aumento do empuxo superficial. Por outro lado, o resfriamento da superfície durante a noite provocou um aumento da densidade superficial, e o consequente aprofundamento e intensificação da turbulência na CMO (Anderson et al., 1996).

O modelo, no dia 14, estimou um valor mínimo de $6 \mathrm{~m}$ na espessura da $\mathrm{CMO}$, coincidindo com um pico de $24,6^{\circ} \mathrm{C}$ na TSM. Porém, neste mesmo dia ocorreu um aprofundamento da ordem de $16 \mathrm{~m}$ associado à entrada da frente fria. A maior produção de energia cinética turbulenta no oceano superior, devido à intensificação do cisalhamento do vento e aumento nos fluxos turbulentos na interface ar-mar, induziu este aprofundamento da CMO (Anderson et al., 1996; Xue et al., 2000). Esse aumento na extensão vertical da CMO foi pequeno se comparado ao aprofundamento de $40 \mathrm{~m}$ observado por Xue et al. (2000) durante a passagem de uma frente fria sobre a Corrente do Golfo.

A camada de mistura se manteve então relativamente mais profunda no período pós-frontal (dia 15 a 20 de Janeiro), chegando a um máximo de $25 \mathrm{~m}$ no dia 18. Assim, mesmo com a diminuição dos ventos nestes dias, a maior perda de calor para

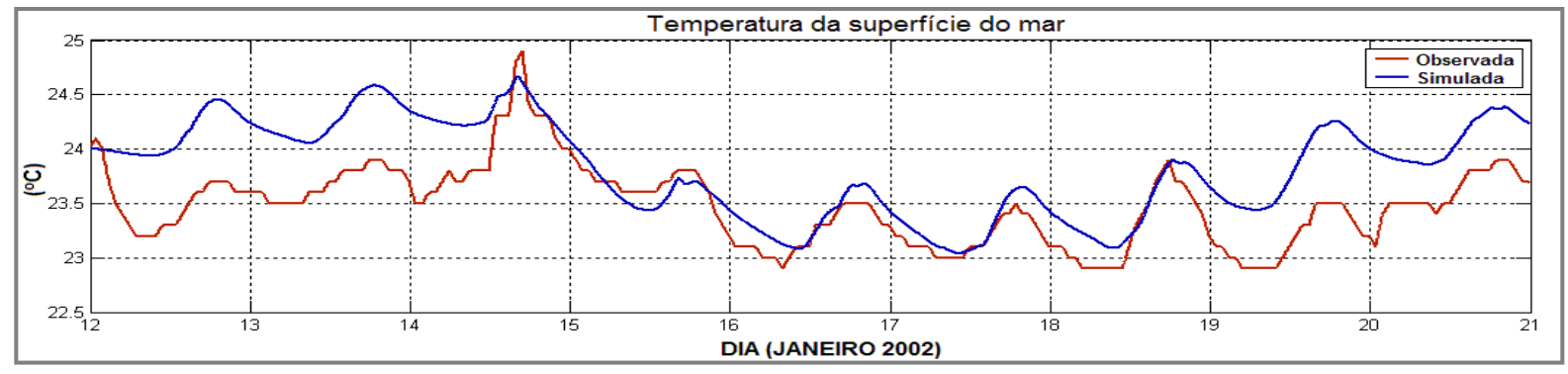

Figura 4 - Evolução temporal da temperatura da superfície do mar para o período de estudo. 
a atmosfera em relação ao período pós-frontal, assegurou que a espessura da CMO se mantivesse próxima a $25 \mathrm{~m}$. Somado a isto, o entranhamento da CMO que promove a injeção de águas mais frias da termoclina no oceano superficial, atuaram também junto aos fluxos de calor para uma menor TSM e maior espessura da CMO, observada até o dia 18.

A partir do dia 18, a extensão da CMO voltou a diminuir gradativamente, em concordância com o aumento da TSM ao final da simulação, na medida em que os fluxos turbulentos de calor reduziram e o oceano e a atmosfera tenderam a um equilíbrio.

As variações observadas para a camada de mistura com a entrada da frente foram acompanhadas também pela camada isotérmica. Isto mostra que a CMO foi controlada, principalmente pela temperatura do oceano superficial, uma vez que a salinidade não variou significativamente no período, já que o balanço de água doce em superfície não foi considerado na simulação.

Os resultados observados aqui, para a evolução temporal da profundidade da $\mathrm{CMO}$, foram semelhantes àqueles encontrados por Dourado e Oliveira (2008). Estes autores, ao investigar o impacto dos contrastes térmicos entre o ar e o mar nas camadas limite atmosférica e oceânica na região de Cabo Frio, verificaram um aprofundamento de até 5,4 $\mathrm{m}$ da CMO associado à passagem de uma frente fria e a interrupção do regime de ressurgência.

\subsection{Balanço de energia}

O balanço de energia sobre a superfície do oceano (Q) foi avaliado no modelo a partir dos fluxos radiativos e turbulentos de calor sensível (H) e latente (LE) na interface ar-mar:

$$
Q=R n+H+L E
$$

Onde Rn é a radiação líquida na superfície do oceano, que é determinada pelo saldo do balanço entre os fluxos radiativos na interface oceano-atmosfera. $\mathrm{O}$ balanço é dado pela expressão $R n=R l \downarrow+R l \uparrow+R c \downarrow+R c \uparrow$ em que $R l \downarrow$ e $R l \uparrow$, são a radiação de onda longa incidente e a emitida pela superfície, respectivamente, $R c \uparrow \quad$ e $\quad R c \downarrow$ são a radiação de onda curta incidente e a refletida pela superfície do mar, respectivamente. A radiação de onda longa, emitida pela atmosfera, foi avaliada no modelo de acordo com a seguinte fórmula empírica para condições de céu nublado (Jacobs, 1978):

$$
O L^{c} \downarrow=O L \downarrow\left(1+0,1762 c^{2}\right)
$$

onde c é o índice cobertura de nuvens, que foi definido como sendo constante e igual a 1 (céu completamente coberto) durante todo o período simulado, e $R l \downarrow$ é a radiação de onda longa emitida pela atmosfera em condição de céu claro, dada por:

$$
R l c \downarrow=\sigma T_{a}^{4}\left(9,365.10^{-6} T_{a}^{2}\right)
$$

onde $\mathrm{T}_{\mathrm{a}}$ é a temperatura do ar e $\sigma=5,67 \times 10^{-8} \mathrm{~W} \cdot \mathrm{m}^{-2} \mathrm{~K}^{-4}$ é a constante de Stefan-Boltzman.

A radiação de onda longa emitida pelo oceano $(R l \uparrow)$ foi estimada por $R l \uparrow=\varepsilon \sigma T S M^{4}$ de acordo com a lei de StefanBoltzman, onde $\varepsilon=0,96$ é emissividade da água e TSM é a temperatura da superfície do mar em Kelvin, considerando-a igual à temperatura equivalente.

Os valores médios dos termos do balanço de energia estimados para o período são apresentados na Tabela 1. Os valores negativos indicam transferência do oceano para atmosfera. Na tabela observa-se que no período pré-frontal a energia líquida armazenada em superfície foi maior em relação aos dias que seguiram a passagem da frente fria. Isto porque, antes da entrada do sistema frontal, houve uma menor perda de energia devido à baixa intensidade dos fluxos de calor latente $\mathrm{e}$ do fluxo líquido de onda longa na superfície.

Antes da passagem frontal a alta umidade relativa do ar, próxima a $80 \%$, e a atuação de ventos da ordem de $4 \mathrm{~m} / \mathrm{s}$, implicaram em uma baixa intensidade do fluxo turbulento de calor latente, que contribuiu para uma menor perda de energia do oceano para a atmosfera (Figura 6). Somado a isto, o fluxo líquido de onda longa na superfície foi menor no período anterior à frente, como resposta de uma maior emissão atmosférica devido à alta umidade e temperatura do ar propiciando assim uma menor perda de energia em relação ao período pós-frontal.

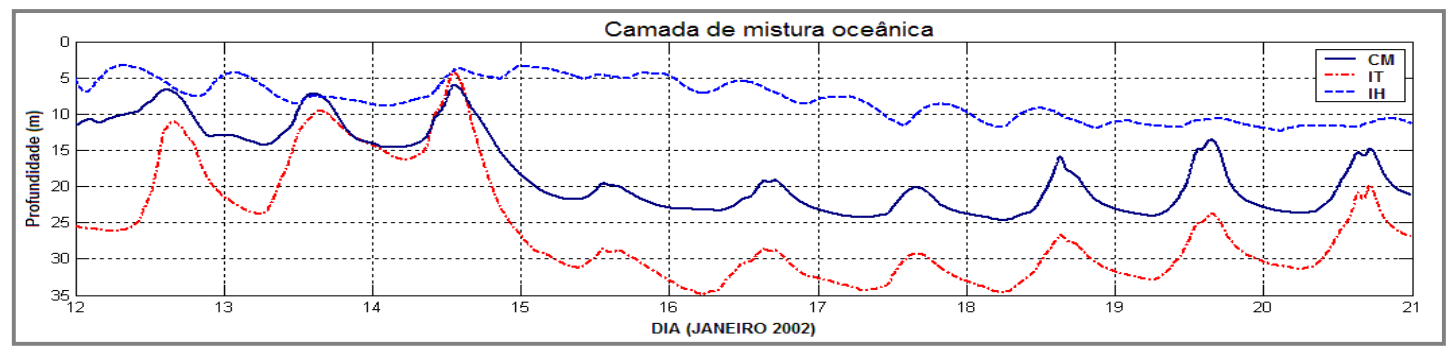

Figura 5 - Evolução temporal da profundidade das camadas de mistura (CM), isotérmica (IT) e isohalina (IH), estimadas para o período de estudo. 
Tabela 1 - Médias (desvio padrão) das componentes do balanço de energia em superfície (Q) estimadas para período de estudo.

\begin{tabular}{lccccc}
\hline & $\mathrm{LE}\left(\mathrm{W} / \mathrm{m}^{2}\right)$ & $\mathrm{H}\left(\mathrm{W} / \mathrm{m}^{2}\right)$ & $\begin{array}{c}R l \uparrow \downarrow \\
\left(\mathrm{W} / \mathrm{m}^{2}\right)\end{array}$ & $R c \uparrow \downarrow$ & $\mathrm{Q}\left(\mathrm{W} / \mathrm{m}^{2}\right)$ \\
\hline Pré-frontal & $-43,57$ & 15,16 & $-16,37(0,96)$ & 280,93 & 236,15 \\
(dias 12 e 13) & $(17,89)$ & $(1,66)$ & & $(326,97)$ & $(327,49)$ \\
Pós-frontal & $-204,1$ & $-9,55$ & $-32,27(5,48)$ & 266,45 & 20,53 \\
(dia 15 a 20) & $(67,33)$ & $(9,99)$ & & $(322,31)$ & $(322,93)$ \\
Todo o período & $-163,82$ & $-3,97$ & $-27,29(8,19)$ & $(316,46)$ & $(351,56)$ \\
\hline dia 12 a 20) & $(101,24)$ & $(14,01)$ & & $(36,11$ & 66,03 \\
\hline
\end{tabular}

NOTA: LE calor latente; H calor sensível; $R l \downarrow \uparrow$ balanço de onda longa; $R c \downarrow \uparrow$ balanço de onda curta.

Com a entrada da frente fria no dia 14, e o consequente aumento da velocidade do vento e redução da umidade relativa do ar, o fluxo de calor latente sofreu uma intensificação da ordem de $-400 \mathrm{~W} / \mathrm{m}^{2}$. Isto porque a atuação de ventos mais intensos leva a um aumento da mistura turbulenta na interface oceanoatmosfera, que intensifica a transferência de momentum do ar para a camada superficial do oceano, levando a uma elevação das taxas de energia liberadas na atmosfera por evaporação (Rogers, 1995). Além disso, a redução na umidade, observada com a entrada da frente fria, também contribuiu para a uma maior taxa de evaporação, devido ao gradiente vertical de umidade no ar (Webster e Lukas, 1992).

O fluxo de calor sensível também atuou para uma maior perda de energia no período pós-frontal. No dia da entrada da frente, o fluxo foi intensificado e, alterando seu sinal, atingiu um pico de aproximadamente $-30 \mathrm{~W} / \mathrm{m}^{2}$. Após a passagem frontal, o fluxo de calor sensível passou a estar direcionado da superfície à atmosfera, em virtude de uma menor $\mathrm{T}_{\mathrm{a}}$ em relação à TSM.

Nos dias seguintes à entrada da frente fria a perda energia pela superfície devido aos fluxos turbulentos de calor $(\mathrm{H}+\mathrm{LE})$, foi da ordem de $-213,57 \mathrm{~W} / \mathrm{m}^{2}$, em contraste com um fluxo médio de $-28,40 \mathrm{~W} / \mathrm{m}^{2}$ no período pré-frontal. No decorrer do período pós-frontal, os fluxos de calor acompanharam as variações da velocidade do vento e da diferença $\mathrm{T}_{\mathrm{a}}$-TSM, e diminuíram lentamente mostrando que, se não houver a entrada de uma nova frente fria ou advecção de massa de água, a superfície do mar e o ar adjacente tendem ao equilíbrio.

Alguns estudos (Tokinaga, et al., 2005, Acevedo et al., 2010) mostraram a importância da variação da TSM na modulação da velocidade do vento na região da Convergência Brasil-Malvinas. Entretanto, como a velocidade do vento é uma forçante externa ao modelo oceânico unidimensional não é possível avaliar um feedback da TSM na velocidade.

O saldo de energia em superfície durante o período foi determinado principalmente pelo balanço entre a radiação de onda curta incidente e o fluxo de calor latente do oceano para a atmosfera. Antes da entrada da frente fria (dias 12 e 13) o balanço entre os fluxos radiativos e turbulentos de calor, contribuía para um ganho de energia, chegando a valores negativos apenas durante o período noturno, onde as perdas radiativas superavam os ganhos. Com a passagem frontal, a intensificação do fluxo de calor latente implicou em uma perda de energia de até -500 $\mathrm{W} / \mathrm{m}^{2}$. As perdas representadas pelos fluxos de calor sensível e de onda longa foram menos significativas.

Esta maior perda de energia no período pós-frontal, dada principalmente pelo fluxo turbulento de calor latente, também foi observada por Persson et al. (2005), que avaliaram processos de interação ar-mar durante a passagem de 10 sistemas frontais sobre uma região do Oceano Atlântico Norte. Eles verificaram que os fluxos de calor sensível e latente foram significativamente maiores na porção pós-frontal do sistema onde houve advecção de uma massa de ar mais fria que a superfície do mar.

\subsection{Temperatura de pele}

A Temperatura da Superfície do Mar é um parâmetro difícil de ser definido exatamente, uma vez que o oceano superior apresenta uma complexa e variável estrutura vertical da temperatura (Donlon et al., 2002). Os fluxos radiativos e os turbulentos de calor, umidade e momentum entre o mar e $\mathrm{o}$ ar influenciam diretamente no calor armazenado no oceano superior, e consequentemente na estrutura da temperatura da superfície (Wick et al., 1996; Donlon et al., 2002).

Na maior parte do tempo, a temperatura de uma camada muito fina na interface oceano-atmosfera (temperatura de pele) é mais fria que a água inferior (temperatura de balde) tipicamente entre 0,1 a $0,5^{\circ} \mathrm{C}$ (Robinson et al., 1984). A diferença resulta do efeito de resfriamento de pele (cool skin effect) em uma camada da ordem de milímetros de espessura na superfície do mar, cuja transferência vertical é dominada por processos moleculares. Durante o dia, o aquecimento do oceano superior pela radiação solar pode resultar na formação de uma camada quente chamada 


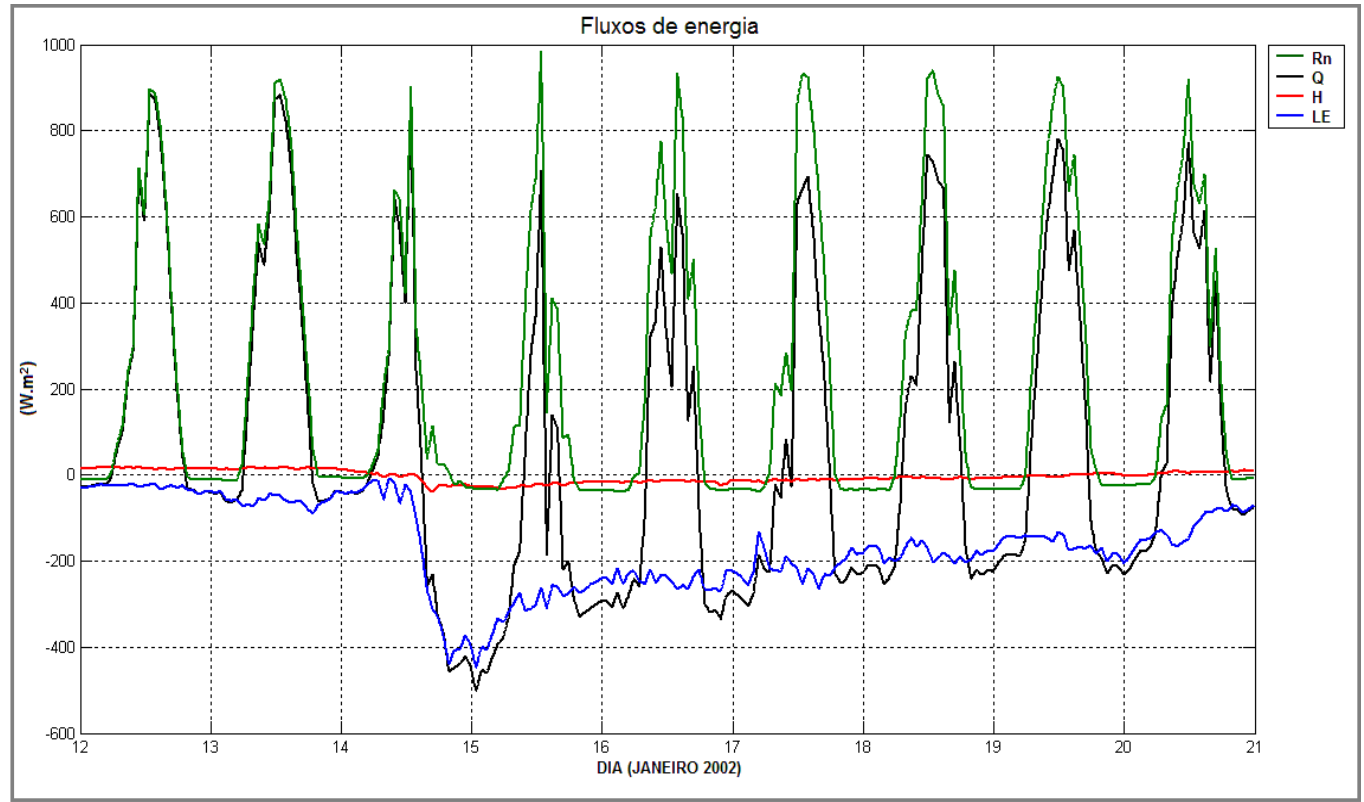

Figura 6 - Evolução temporal dos fluxos de energia estimados para o período de estudo. Rn é a radiação liquida, Q é o balanço de energia líquida, H é o fluxo de calor sensível e LE o fluxo de calor latente em $(\mathrm{W} / \mathrm{m} 2)$.

warm layer, que se opõe ao efeito cool skin (Fairral et al., 1996; Dourado e Caltabiano, 2005).

O gradiente térmico vertical na camada superficial do oceano interfere na definição correta da TSM, podendo variar de acordo com o método utilizado para a sua obtenção. As medidas utilizando boias são feitas, geralmente em profundidades próximas de $1 \mathrm{~m}$, como é o caso da boia utilizada neste trabalho, que registrou os valores de temperatura em $0,5 \mathrm{~m}$ de profundidade. Dessa forma, esses valores de TSM diferem daquela obtidos por satélites, por exemplo, que registram medidas na camada superior ( $10 \mu \mathrm{m}$ e $12 \mu \mathrm{m})$ e considera o efeito da camada de pele (Dourado e Caltabiano, 2005).

No presente estudo, a temperatura de pele foi obtida a partir da temperatura de balde medida pela boia, tomando em conta as correções da cool skin e warm layer, estimadas por um esquema de camada de pele acoplado ao modelo (Fairall et al., 1996). Assim, a estimativa foi feita da seguinte forma:

$$
T_{\text {pele }}=T_{\text {balde }}-\Delta T c+\Delta T w
$$

onde $\Delta T c$ é a correção da cool skine $\Delta T w$ a da warm layer.

Para o período pré-frontal, a temperatura de pele estimada foi superior à temperatura de balde (Figura 7a). Neste período, dois fatores são importantes para isto. Nos dias $12 \mathrm{e}$ 13 , os ventos em torno de $10 \mathrm{~m} / \mathrm{s}$ misturam a superfície do mar, tornando esta camada mais homogênea. No dia 14, os ventos diminuem e o ciclo diurno da radiação solar foi suficiente para reduzir significativamente o gradiente de temperatura da camada de pele fria (Figura 7b).
Em oposição, o aquecimento intensificou a camada quente (Figura 7c), fazendo com que a Tpele atingisse valores superiores à Tbalde, em até $0,49^{\circ} \mathrm{C}$. Esta intensificação da warm layer foi mais significativa no dia 14 , chegando a um máximo de $0,48^{\circ} \mathrm{C}$, devido aos valores do fluxo de calor latente terem oscilado próximos a zero neste dia. Por esse motivo foi encontrado o valor máximo de $25,3^{\circ} \mathrm{C}$ na Tpele.

Por outro lado, após a passagem da frente fria (dia 15 a 20), a warm layer mesmo ainda apresentando um ciclo diário, diminuiu o seu gradiente vertical de temperatura. $\mathrm{O}$ aumento verificado pelos fluxos turbulentos de calor latente e sensível direcionados para a atmosfera induziu a um resfriamento na interface e assim, intensificou o efeito da cool skin no período pós-frontal. Somado a isso, a redução na velocidade do vento observada nesse período, também favoreceu este aumento da diferença de temperatura da cool skin. Isso porque, a turbulência gerada por ventos moderados em superfície, é suficiente para dirigir os fluxos turbulentos de calor, sem destruir o efeito do resfriamento de pele (Donlon e Robinson, 1997; Dourado e Caltabiano, 2005).

A estimativa dos fluxos de calor via fórmulas bulk pode variar significativamente, quando se considera a temperatura de pele, já que a TSM é utilizada diretamente no cálculo (Webster e Lukas,1992). A Tabela 2 mostra a influência da inclusão do efeito de resfriamento de pele e da camada quente nos fluxos turbulentos, estimados pelo esquema COARE acoplado ao modelo. A exclusão da cool skin aumentou em $4,47 \mathrm{~W} / \mathrm{m}^{2}$, em média, o fluxo negativo de calor latente e $1,51 \mathrm{~W} / \mathrm{m}^{2}$ o de calor 




(b) Cool skin

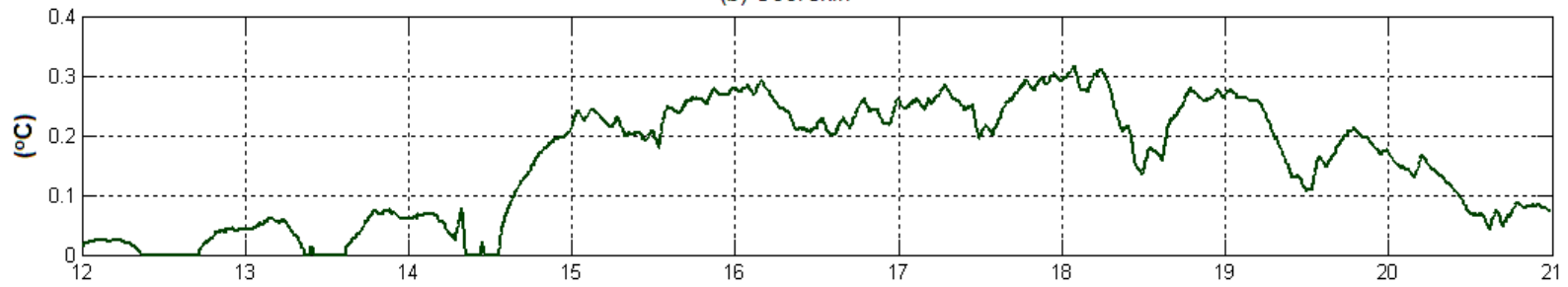

(c) Warm layer

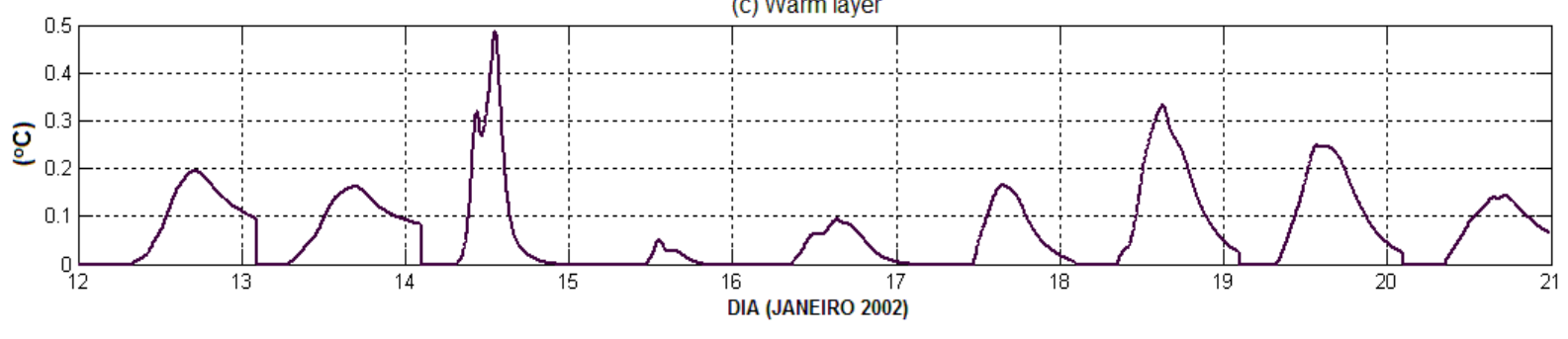

Figura 7 - evolução temporal da (a) temperatura de pele e temperatura de balde, (b) cool skin e (c) warm layer, estimadas para o período de estudo.

Tabela 2 - Fluxos de calor médios (desvio padrão) estimados para o período.

\begin{tabular}{|c|c|c|}
\hline & Calor latente $\left(\mathrm{W} \cdot \mathrm{m}^{-2}\right)$ & Calor sensível $\left(\mathrm{W} \cdot \mathrm{m}^{-2}\right)$ \\
\hline Cool e warm layer & $-173,96(94,05)$ & $-5,61(11,47)$ \\
\hline $\begin{array}{c}\text { Sem a } \\
\text { cool skin }\end{array}$ & $-178,43(98,49)$ & $-7,12(12,45)$ \\
\hline Sem warm layer & $-173,90(94,09)$ & $-5,59(11,48)$ \\
\hline
\end{tabular}

sensível. Este aumento representou uma redução de $6 \mathrm{~W} / \mathrm{m}^{2}$, em média, no calor disponível para aquecer o oceano, que representa $10 \%$ do balanço de energia médio estimado para todo o período.

Já a remoção do efeito da warm layer não levou a uma variação significativa. Isto mostra que o efeito de resfriamento de pele foi significativo durante o período, e sua influência na diferença $T_{a}$-TSM afetou na estimativa dos fluxos turbulentos.

\section{CONCLUSÕES}

A resposta do oceano superficial à passagem de uma frente fria, entre os dias 12 e 20 de Janeiro de 2002, foi investigada na região da Confluência Brasil-Malvinas, utilizando um modelo oceânico unidimensional de camada limite baseado em Mellor e Yamada (Dourado e Oliveira, 2008).

Os resultados da simulação mostraram que o modelo reproduziu bem, tanto o ciclo diurno, quanto a queda de aproximadamente $1^{\circ} \mathrm{C}$ observada da TSM devido à passagem da frente fria. A entrada desse sistema meteorológico no dia 14 foi caracterizada pelo aumento na velocidade do vento e uma intensificação nos fluxos de calor e momento, inclusive com alteração no fluxo de calor sensível relacionada à entrada de uma massa de ar mais fria que a TSM em até $2{ }^{\circ} \mathrm{C}$. Assim, os resultados evidenciaram que o efeito do cisalhamento do vento tem influência em um aumento da mistura turbulenta na interface oceano-atmosfera, que aliada à baixa umidade e temperatura do ar, induziu um aumento nos fluxos turbulentos de calor do oceano à atmosfera. 
A intensificação nos fluxos e uma diminuição na radiação líquida provocou uma alteração no balanço de energia em superfície. Esta perda líquida de até $-500 \mathrm{~W} / \mathrm{m}^{2}$ de energia em superfície, no período pós-frontal, levou a uma modificação na estrutura do oceano superior. O efeito da cool skin foi intensificado, uma vez que a camada de pele responde quase que instantaneamente às variações da turbulência e do fluxo de calor (Schlüssel et al., 1990; Donlon e Robinson, 1997; Dourado e Caltabiano, 2005). O contrário ocorreu com warm layer, que apesar do fluxo solar incidente ainda ter controlado suas variações diurnas, a amplitude destas oscilações foi menor no período pós-frontal, em resposta à menor quantidade de energia armazenada em superfície.

A variação no balanço de energia na interface ar-mar também afetou na extensão da camada de mistura oceânica, que sofreu um aumento de aproximadamente $15 \mathrm{~m}$ em sua extensão. $\mathrm{O}$ aprofundamento foi modulado, em parte pela produção dinâmica de energia cinética turbulenta, devido ao cisalhamento do vento, bem como, pela forçante térmica associada à intensificação dos fluxos turbulentos na interface ar-mar.

\section{AGRADECIMENTOS}

Os autores agradecem ao $\mathrm{CNPq}$, processo individual 475708/2007-5, ao Instituto Nacional de Meteorologia (INMET), a Diretoria de Hidrografia e Navegação (DHN) da Marinha do Brasil e ao Centro de Estudos do Mar da Universidade Federal do Paraná (CEM-UFPR).

\section{REFERENCIAS BIBLIOGRAFICAS}

ACEVEDO, O. C.; PEZZI, L. P.; SOUZA, R. B.; ANABOR, V.; DEGRAZIA, G. A. A. Atmospheric Boundary Layer Adjustment the Synoptic Cycle at the Brazil-Malvinas Confluence, South Atlantic Ocean. Journal of Geophysical Research, v. 115, p. 1-12, 2010.

ANDERSON, S. P.; WELLER, R. A.; LUKAS R. Surface buoyancy forcing and the mixed layer of the western equatorial Pacific warm pool: Observations and 1D model results, Journal of Climate, v. 9, p. 3056-3085, 1996.

ANDRÉ, J. C. ; LACARRÈRE, P. Mean and turbulent structures of the oceanic surface layer as determined from onedimensional third order simulations. Journal of Physical Oceanography, v. 15, p. 121-132, 1985.

BLACKADAR, A. K. The vertical distribution of wind and turbulence exchange in a neutral atmosphere. Journal of Geophysics Research, v. 67, p. 3095-3102, 1962.

D’ALESSIO, S.J.D.; ABDELLA, K.; MC FARLANE, N.A. A new second-order turbulence scheme for modeling the ocean mixed layer. Journal of Physical Oceanography, v. 28, p. 1624-1641, 1998.
DONLON C. J.; ROBINSON, E I.S. Observations of the oceanic thermal skin in the Atlantic. Journal of Geophysics Research, v. 102, p. 18585-18606, 1997.

DONLON, C. J.; MINNETT, P.J.; GENTEMANN, C.; NIGTINGALE, T.J.; BARTON, I.J.; WARD, B.; MURRAY, M.J. Toward Improved Validation of Satellite Sea Surface Skin Temperature Measurements for Climate Research, Journal of Climate, v. 15, p. 353-369, 2002.

DOURADO, M.S.; CALTABIANO, A.C.V. Novos Conceitos na Definição da Temperatura da superfície do Mar. In: SOUZA, R. B. (Org.). Oceanografia por Satélites. 1 ed. São Paulo: Oficina de Textos, n. 1, p. 136-147, 2005.

DOURADO, M.S.; CANIAUX, G. Surface heat budget in an oceanic simulation using data from Tropical OceanGlobal Atmosphere Coupled Ocean-Atmosphere Response Experiment. Journal of Geophysics Research, v. 106, p. 623-640. 2003.

DOURADO, M. S.; OLIVEIRA, A.P. de. Observational Description of the Atmospheric and Oceanic Boundary Layers over the Atlantic Ocean. Revista Brasileira de Oceanografia, v. 49, p. 49-64, 2001.

DOURADO, M.S.; OLIVEIRA, A.P. de. A numerical investigation of atmosphere-ocean thermal contrast on the PBL shor-term variation over the coastal upwelling region of Cabo Frio, Brazil. Atmosféra, v. 21, p. 13-34, 2008.

FAIRALL, C.W.; BRADLEY, E.F.; ROGERS, D.P.; EDSON, J.B.; YOUNG, G.S. Bulk parameterization of air-sea fluxes for Tropical Ocean-Global Atmosphere Coupled-Ocean Atmosphere Response Experiment. Journal of Geophysics Research, v. 101, p. 3747-3764, 1996.

FAIRALL, C.W.; BRADLEY, E.F. Bulk Parameterization of air-Sea Fluxes: Updates and Verification for the COARE algorithm. Journal of Climate, v. 16, p. 571-591, 2003.

GASPAR, P; GREGORIS, Y.; LEFEVRE, J.M. A simple eddy kinetic energy model for simulations of the oceanic vertical mixing: Test at station Papa and long term upper ocean study site. Journal of Geophysical Research, v. 95, p. 16179-16193, 1990.

JACOBS, J. D. Radiation climate of Broughton Island. Energy budget studies in relation to fast-ice breakup processes in Davis Strait. Institute of Arctic and Alpine Research, University of Colorado, Boulder, United States, v.26, p.105120, 1978.

KHANTA, L.H.; CLAYSON, C.A. An improved mixed layer model for geophysical applications. Journal of Geophysical Research, v. 99, p. 25235 - 25266, 1994.

MELLOR, G. L.; YAMADA, T. A hierarchy of turbulence closure models for planetary boundary layers. Journal of the Atmospheric Sciences, v. 31, p. 1791-1806, 1974.

MELLOR, G. L.; YAMADA, T. Development of a turbulence closure model for geophysical fluid problems. Reviews of Geophysics and Space Physics, v. 20, p. 851-875, 1982. 
PERSSON, G.P.O.; HARE, J.E.; FAIRALL, C.W.; OTTO, W.D. Air-sea interaction processes in warm and cold sectors of extratropical cyclonic storms observed during FASTEX. Quaterly Journal of the Royal Meteorological Society, v.131, p. 877-91, 2005.

PEZZI, L. P.; SOUZA, R. B.; DOURADO, M. S.; GARCIA, A. E.; MATA. M. M. Ocean- Atmosphere in situ observations at the Brazil-Malvinas confluence region. Geophysical Research Letters, v. 32, 10.1029/2005GL023866, 2005.

PIOLA, A. R.; CAMPOS, E. J. D.; MÖLLER, O. O.; CHARO, M.; MARTINEZ, C. Subtropical Shelf Front off eastern South America. Journal Geophysical Research, v. 105, p. 6565-6578, 2000.

PIOLA, A. R.; MATANO, R. P. Brazil and Falklands (Malvinas) currents. In: THORPE, S. A. Encyclopedia of Ocean Sciences, p. 340- 349, Elsevier, New York, 2001.

ROBINSON, I.S.; WELLS, N.C.; CHARNOCK, H. The sea surface thermal boundary layer and its relevance to the measurement of sea surface temperature by airborne and spaceborne radiometers, International Journal of Remote Sensing, v. 5, p. 19-45, 1984.

RODRIGUES, M.L.G.; FRANCO, D.; SUGAHARA, S. Climatologia de frentes frias no litoral de Santa Catarina. Revista Brasileira de Geofísica, São Paulo, v. 22, n. 2, 2004.

ROGERS, D. P. Air sea interaction: connecting the ocean and atmosphere. Scripps Institution of Oceanography, La Jolla, California. Reviews of Geophysics, v. 33, Suppl. American Geophysical Union, 1995.

SCHLUESSEL P.; EMERY, W.J.; GRASSL, H.; MAMMEN, T. On the bulk-skin temperature difference and its impact on satellite remote sensing of sea surface temperature. Journal of Geophysical Research, v. 95, p. 13341-13356, 1990.

SILVEIRA, I.C.A.; SCHMIDT, A. K.; CAMPOS, E.J.D. A Corrente do Brasil ao Largo da Costa Leste Brasileira. Brazilian Journal of Oceanography, n.2, v. 48, p.171183, 2000.
SOARES, I.; MÖLLER, O. O. Low-frequency currents and water mass spatial distribution on the southern Brazilian shelf. Continental Shelf Research, v. 21, p. 1785-1814, 2001.

SPRINTALL, J.; TOMCZAK, M. Evidence of the barrier layer in the surface layer of the Tropics. Journal of Geophysical Research, v. 97, p. 7305-7316, 1992.

TOKINAGA, H.; TANIMOTO, Y.; XIE, S.-P. SST-Induced Surface Wind Variations over the Brazil-Malvinas Confluence: Satellite and In Situ Observations. Journal of Climate, v. 18, p. 3470-3482, 2005.

XUE, H.; PAN, Z.; BANE, J.M. A 2D coupled atmosphereocean model Study of air-sea interactions during a cold air advection over the Gulf Stream. Monthly Weather Review, v. 128, p. 973-966, 2000.

WALLACE, J.M.; HOBBS, P.V. Atmospheric Science: An Introductory Survey. 2 ed., London Academic Press, 2006, $483 \mathrm{p}$.

WEBSTER, P. J.; LUKAS, R. TOGA COARE: The coupled ocean-atmosphere response experiment. Bulletin of American Meteorological Society, v. 73, n. 9, p. 13771416, 1992.

WENG W.; TAYLOR P. On modeling the one-dimensional atmospheric boundary layer. Boundary-Layer Meteorology, v. 107, p 371-400, 2003.

WICK, G. A., EMERY, W. J.; KANTHA, L. H.; SCHLUESSEL, P. The behavior of the bulk-skin sea surface temperature difference under varying wind speed and heat flux, Journal of Physical Oceanography, v. 26, p. 1969-1988, 1996. 